\title{
Investigation of Pellet-Triggered MHD Events in the ASDEX Upgrade ${ }^{1}$
}

\author{
P. T. Lang, K. Lackner, A. Kallenbach, M. Maraschek, C. P. Perez von Thun, W. Suttrop, \\ and the ASDEX Upgrade Team \\ Max-Planck-Institut für Plasmaphysik, EURATOM Association, Boltzmannstr. 2, 85748 Garching, Germany
}

Received December 24, 2007

\begin{abstract}
In order to gain deeper insight into the process of MHD activity triggered by pellets we extended our previous analysis (standard type-I edge localized modes (ELMs)) to type-I ELMs in radiative edge scenarios, type-III ELMs, the quiescent H-mode regime, and core mode activity such as neoclassical tearing modes or snakes. Pellet triggering of mode activity has turned out to be a quite general feature, but only in case of the ELMs can it be unambiguously attributed to prompt local impact by the pellet. For edge plasma conditions characterized by higher resistivity, the growth time of spontaneous ELMs increases while the plasma changes from the type-I into the type-III regime. However, pellet-triggered ELMs retain fast rise times. In the quiescent Hmode, pellets still trigger ELM-like mode activity, but no longer accompanied by a significant release of energy from the plasma.
\end{abstract}

PACS numbers: 52.35.Py, 52.55.Fa, 52.55.Tn

DOI: $10.1134 / \mathrm{S} 1063780 \mathrm{X} 08090018$

\section{INTRODUCTION}

Edge-localized-mode-(ELM-) -induced fast erosion of plasma-facing materials has been recognized as one of the greatest threats to the viability of ITER. Several approaches to solving this problem are currently under investigation. One possible option is to establish ELM control using the concept of pacing. The idea is to raise the controlled ELM frequency, $f_{\mathrm{ELM}}$, above the natural ELM frequency, $f_{\mathrm{ELM}}^{0}$, and thus reduce the energy per ELM. Proof-of-principle experiments performed at ASDEX Upgrade [1] demonstrated the feasibility of the approach for midsize tokamaks by pacing via injection of cryogenic millimeter-sized pellets. Here, we broaden the triggering investigations beyond the standard scenario type-I ELM regime in order to gain deeper insight to the ELM triggering process and the related dynamics of imposed perturbation and ELM growth. We picked two scenarios with enhanced edge resistivity, the type-I regime with a cooled radiative edge shell established by Argon puffing and the type-III regime achieved by edge cooling via gas puffing. In addition, pellet injection during quiescent $\mathrm{H}$-mode $(\mathrm{QH})$ phases, characterized by absence of spontaneous ELMs, was investigated. In addition to their impact on edge modes, we revisited as well investigations regarding pellet triggering of core MHD modes. The examples chosen here were neoclassical tearing modes (NTMs) and the $q=1$ snake.

\footnotetext{
${ }^{1}$ The text was submitted by the authors in English.
}

It should be noted that the analysis presented here is based on already-available data. Hence, most results are somewhat hampered by less than fully optimized pellet and/or diagnostics settings. Dedicated experiments are underway and will be the subject of future reports.

\section{EXPERIMENTAL SETUP}

The experiments to which we refer to have been performed in ASDEX Upgrade, a midsized diverter tokamak with torus radius $R_{0}=1.65 \mathrm{~m}$, minor plasma radius $a_{0}=0.5 \mathrm{~m}$, typical plasma elongation $b / a>1.6$, and plasma volume $V_{\text {plasma }}=13 \mathrm{~m}^{3}$ [2]. The diverter geometry and wall material composition have undergone several revisions. For a description of the particular setup and plasma configurations, we refer to the corresponding documentation on the main experiments performed. The pellet injector employed is based on a centrifuge pellet accelerator equipped with a storage cryostat pellet reservoir. It is capable of delivering up to 120 cubic pellets of nominal size $(1.4 \mathrm{~mm})^{3}-(1.9 \mathrm{~mm})^{3}$ (nominal pellet masses $m_{p}$ from $1.6 \times 10^{20}$ to $4.1 \times$ $10^{20} \mathrm{D}$-atoms)) at velocities $V_{p}$ from 240 to $1200 \mathrm{~m} / \mathrm{s}$ and repetition rates up to $80 \mathrm{~Hz}$. Again, several modifications of the injection geometry have taken place, but information on the precise version used for a specific experiment can be found in the references provided. 


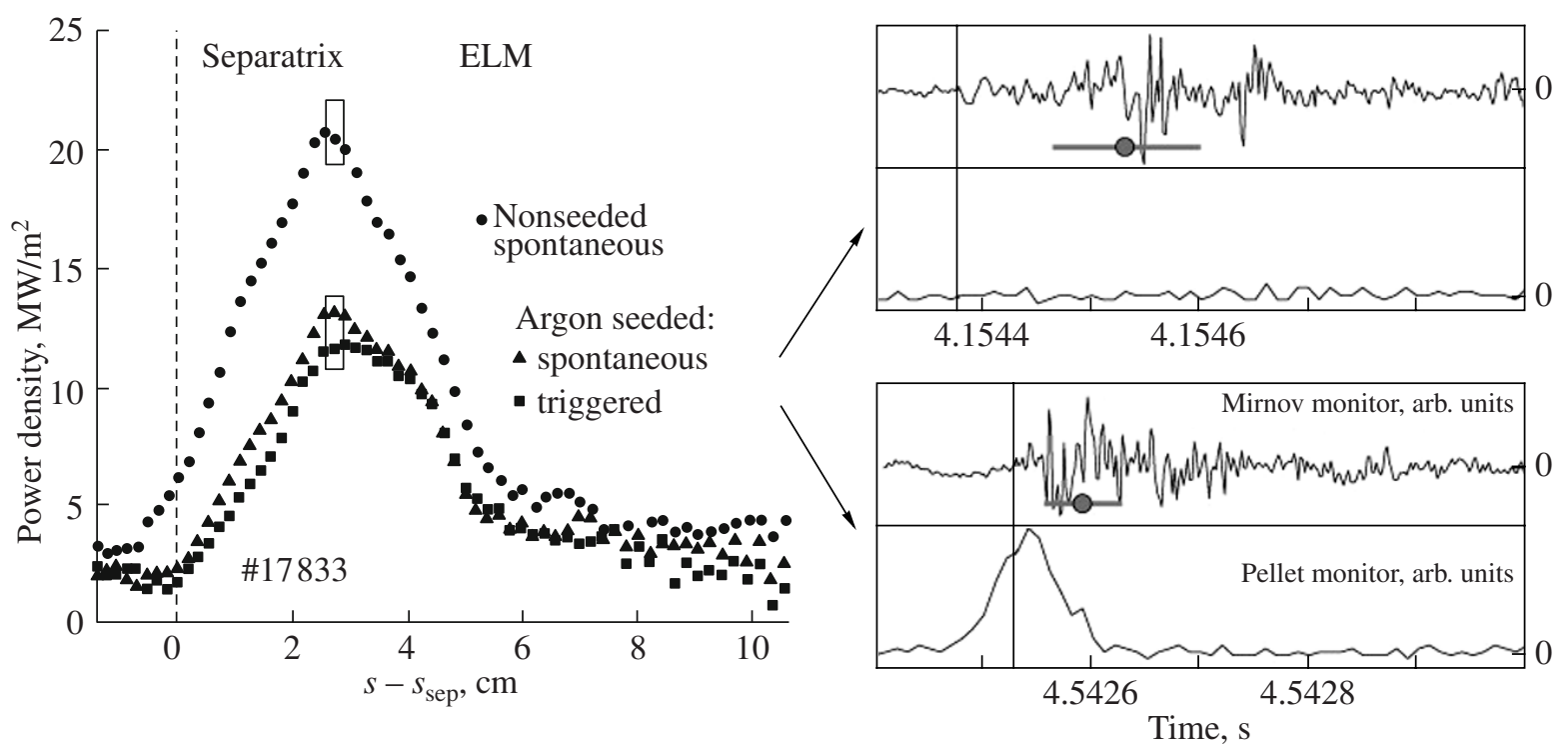

Fig. 1. Comparison of spontaneous and triggered type-I ELMs in radiative cooled-edge phases. Whereas there is no difference in their global action - e.g., the imposed heat load (left) - the onset dynamics, as characterized by the mode amplitude (right), is different. Pellet-triggered events show significantly faster growth to maximum amplitude $(60 \pm 36 \mu \mathrm{s}$ for the entire sample as indicated) than their spontaneous counterparts $(154 \pm 68 \mu \mathrm{s})$. Ar seeding results in a significant heat flux reduction on the diverter (left, boxes representing the standard deviation of entire sample of events).

\section{RESULTS}

\subsection{Standard (Hot-Edge) Type-I Regime}

The current ITER baseline scenario is a H-mode with hot edge and clear type-I ELMs. Uncontrolled, these large ELM bursts could reduce the diverter lifetime considerably [3]. Hence, this regime has already been well investigated both in pacing and perturbative approaches. Details have been reported in a recent study [4], though the main results are briefly summarized here for convenience. No significant difference can be observed between triggered and spontaneous ELMs, with respect to neither their global appearance as characterized by energy loss and diverter power flux nor their dynamics-if the same repetition frequencies are considered. The perturbation forming the trigger is imposed in the center of the transport barrier, and a delay of about $50 \mu$ s between perturbation deposition and ELM onset is observed. No significant spatial relation between the poloidal and toroidal location of first ELM indication and pellet injection position can be found. The ELM MHD signature then becomes visible in the entire edge region within about $20 \mu \mathrm{s}$. (For reference, the fast shear Alfvén wave transit time $v_{A} / 2 \pi R_{0} \approx$ $1 \mu \mathrm{s}$.) Between the first indication and maximum amplitude of the corresponding MHD-induced $d B / d t$ signal, fast growth is observed. For an ensemble of spontaneous ELMs for this growth time, a value of $67 \pm$ $22 \mu$ s was derived, an ensemble of triggered ELMs under identical plasma conditions delivering a virtually identical value of $72 \pm 22 \mu \mathrm{s}$.

\subsection{Radiative (Cool-Edge) Resistive Type-I Regime}

The experiment on controlled edge-and, hence, diverter-cooling by Ar seeding is reported in [5]. During the approach pellet pacing was applied to avoid ELM free phases. However, the resulting ELM frequency became significantly higher than the pellet pacing frequency. Consequently, during pacing periods, both spontaneous and triggered ELMs occur. No significant difference is found for their global action, all along the Ar seeding phase a constant reduction of the deposited power density along strike point contours is found. Following Kallenbach's analysis [5], the power load close to the outer strike point was investigated, as is shown in Fig. 1 (left). Triggered and spontaneous ELMs showed identical behavior within individual scatter (boxes representing the $2 \sigma$ width), but reasonable reduction with respect to the nonseeded reference. The two types differ, however, in onset dynamics, as shown in the right part of Fig. 1 (signal traces of typical single events and averages for entire ensembles). Whereas spontaneous ELMs grow significantly more slowly in the cool-edge regime (154 $\pm 68 \mu \mathrm{s})$, triggered ones $(60 \pm 38 \mu \mathrm{s})$ retain the fast onset observed in the hot-edge case. This indicates that triggering can restore fast ELM growth in the resistive edge scenario with its otherwise reduced growth rates for spontaneous ELMs.

\subsection{Cool-Edge Resistive Type-III Regime}

Increasing the edge resistivity further by, e.g., cooling via strong gas bleeding results in a transition to the type-III ELM regime. A description of this regime can be found in [6], where the transition is attributed to a 


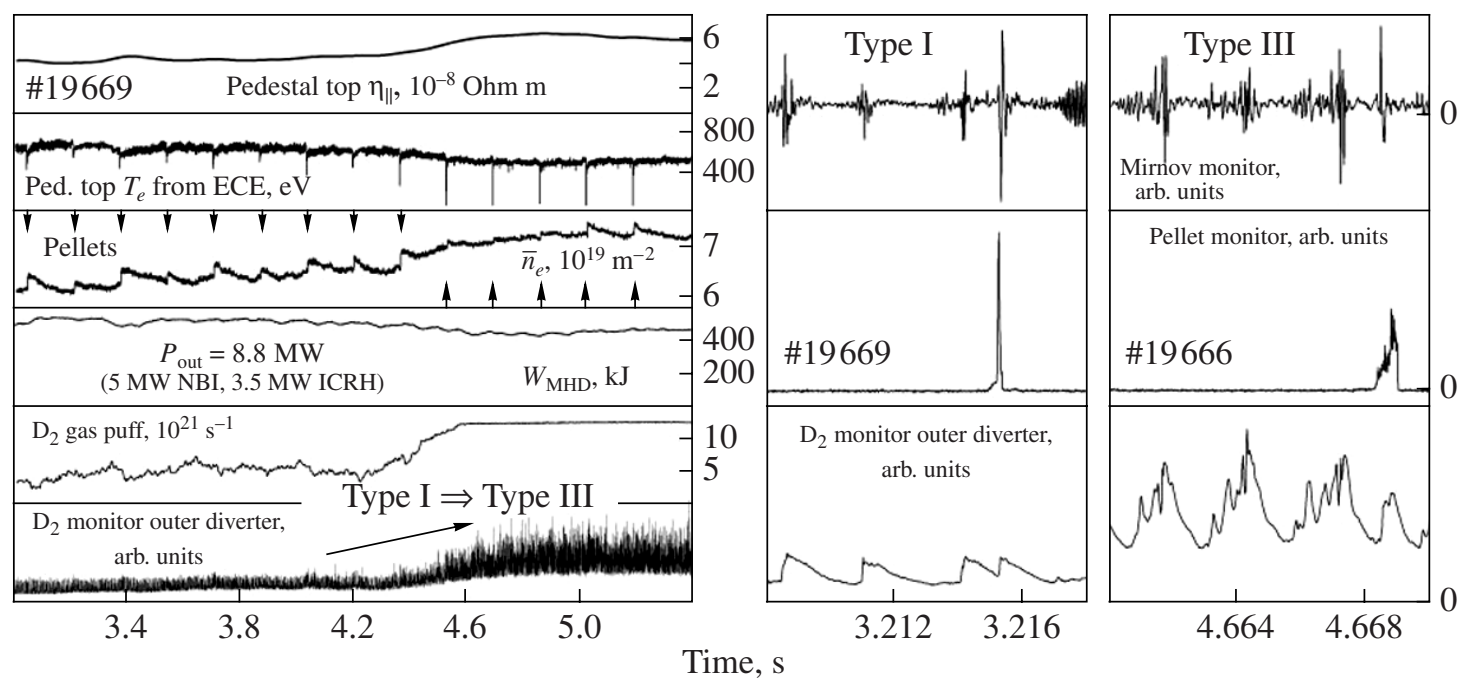

Fig. 2. Edge cooling by gas puffing increases the edge resistivity (parallel Spitzer taking electron data and assuming $Z_{\mathrm{eff}}=1$ ) and causes a transition from the type-I into the type-III regime (left). In the type-I regime (middle), triggered and spontaneous ELMs appear virtually identical. In the type-III regime (right, data from virtually identical shot), spontaneous ELMs evolve more slowly toward maximum amplitude, while triggering seems to restore the type-I onset characteristics.

reduced power flux through the separatrix. The evolution of a discharge driven from type-I into type-III by gas puffing is shown in Fig. 2 (left); the type-III character is confirmed by the frequency of an MHD ELM precursor $(\approx 20 \mathrm{kHz})$ and an inverted relationship between heating power and $f_{\mathrm{ELM}}[6]$. Low-frequency injection of rather large pellets was performed through the transition. During the type-I phase, both spontaneous and triggered ELMs show a fast onset, as can be seen from the burstlike MHD signature and fast-rising $D_{\alpha}$ radiation from the outer strike point region. It should be noted the triggered ELM is somewhat oversized due to the large transient particle and energy throughput connected with pellet ablation-an effect that vanishes as pellet masses drop [1]. In the type-III regime, spontaneous ELMs display a significantly slower evolution. Fast strong type-I-like events are triggered even in this regime by pellets (data shown taken from twin discharge). The event shown corresponds to the situation that a pellet arrives in a truly quiescent phase between subsequent type III ELMs: due to the higher natural frequency and the longer duration, such a clear separation between triggered and spontaneous ELMs - generally the case in type I regimes-occurs only rarely here. Detailed dedicated investigations in this regime are under way to clarify the nature of triggered ELMs using small pellets. Nevertheless, results achieved so far indicate in high edge resistivity type-III regime with slow ELM growth and precursor activity pellet triggering still causes fast type-I-like ELM growth.

\subsection{Quiescent H-Mode Phases}

The $\mathrm{QH}$ mode is a quite interesting regime with respect to edge instabilities and related energy bursts. It no longer displays strong burstlike transport, but an edge harmonic oscillation (EHO). While the $\mathrm{QH}$ regime's very narrow operational range is a clear disadvantage, it is nevertheless of interest to investigate the pellet impact. The experimental aim was to fuel $\mathrm{QH}$ plasmas using pellets; details are reported in [7]. Consequently, the pellet size was chosen to be rather large. The evolution of a discharge showing a transition from type-I to QH without significant changes of global plasma parameters is plotted in Fig. 3 (left). The blowup at the top shows a typical EHO with a fundamental frequency of about $10 \mathrm{kHz}$. On the right part of Fig. 3, a comparison of a spontaneous type-I ELM and the pellet impact in $\mathrm{QH}$ is displayed. The pellet triggers a magnetic perturbation, but, despite the large pellet size, it far from reaches the magnitude of a type-I ELM. Instead, the magnetic signature is rather similar to that following a pellet injection into $\mathrm{OH}$ or L-mode plasmas. Furthermore, there is no type-I like energy release. The slight out flux observed can be attributed to the rather massive perturbation of the pellet and following transport-induced particle losses. Hence, the experimental finding is a pellet-triggered type-I-like MHD activity, but this mode obviously does not "mature" into a fully developed ELM. Rather, it rather seems that the seed perturbation created by the pellet does not face sufficient growth conditions.

\subsection{Core MHD Activity}

Obviously, local 3D perturbations created by pellets can trigger ELMs. For a further broadening of the analysis, the question was raised as to whether this is a specific or a general feature for MHD modes. Therefore, 


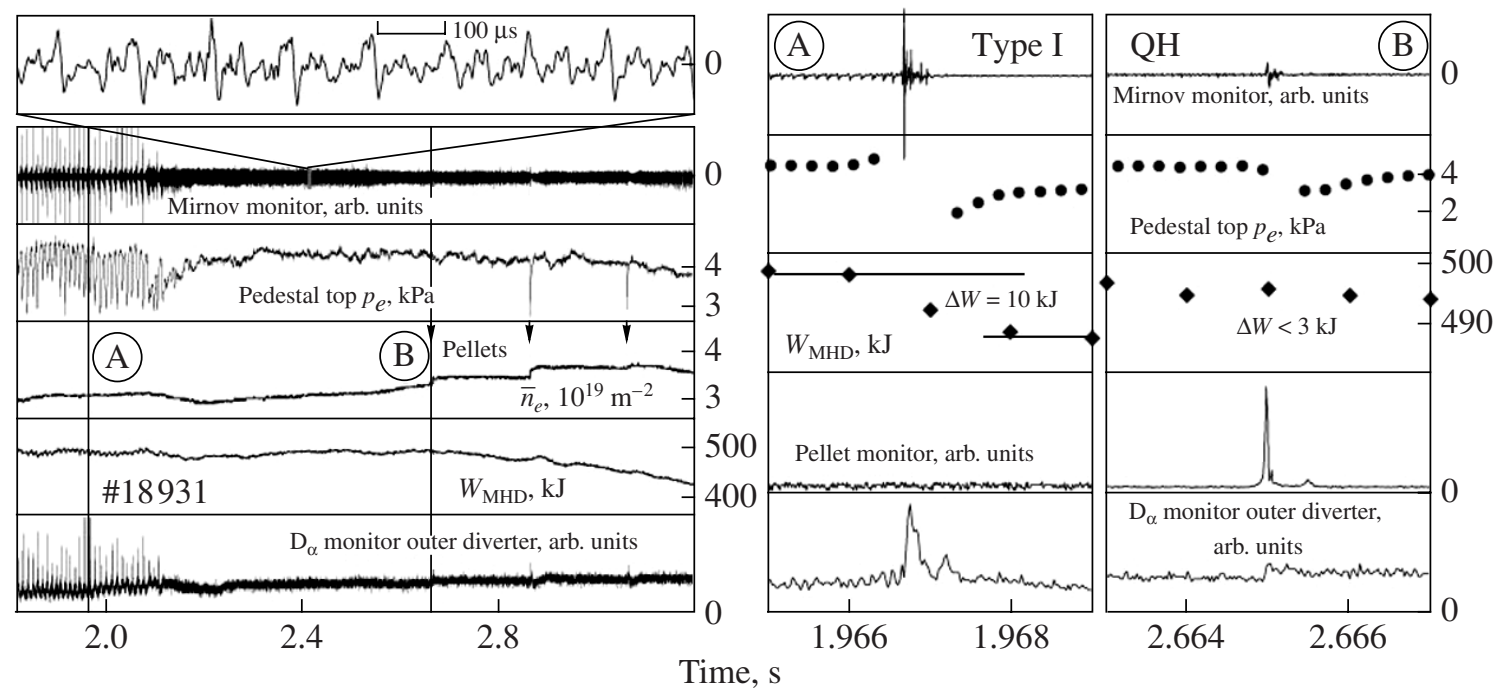

Fig. 3. Transition from type-I ELM regime into a QH phase while keeping plasma parameters virtually identical (left). Burstlike ELM events are replaced by an EHO at about $10 \mathrm{kHz}$ (blowup on top). A pellet injected during the QH phase (right, indicated by (B) in the left part) triggers MHD activity similar to a type-I ELM (middle, (A)) but with less strength. In contrast to the ELM, no significant energy is released during this event.

we revisited two well-documented cases of pellet impact on core MHD modes.

3.5.1. Neoclassical tearing modes. Pellet triggering of NTM was found to hamper the performance in fuelling studies, but turned out to be useful for mapping out onset conditions. A study by Zohm et al. [8] recognized the onset pressure of $m=3, n=2$ NTM as a pure function of local ion temperature by sawtooth or pellet-created seed islands. For both triggers, mode growth lasts several $10 \mathrm{~ms}$. Growing on a transport time scale does not allow one to disentangle whether the trigger was imposed by a local 3D perturbation or global change of plasma parameters at the relevant surface in the pellet case. Moreover, our new analysis indicates that pellet triggering can be achieved without pellet penetration reaching the $q=1.5$ surface.

3.5.2. Snakes at the $q=1$ surface. A phenomenon called snake is attributed to the formation of a helical region with enhanced confinement mostly at the $q=1$ surface under certain conditions. Reliable triggering of such snakes was reported for deep pellet penetration in JET [9] and also observed in ASDEX Upgrade. Again, it is still unclear if pellet perturbation towards the $q=1$ surface is a necessary prerequisite, and again mode growth takes place on the transport time domain of several $10 \mathrm{~ms}$. Pellet triggering of the snake onset confirms NTM results: provided onset conditions are fulfilled the pellet perturbation triggers the event. However, initiation by the prompt local perturbation cannot be proven and a local trigger mechanism is not demonstrated.

\section{CONCLUSIONS}

The investigations presented extend our previous work and broaden the phenomenology of pelletinduced MHD. It can serve as an experimental guideline for investigations aiming on physics interpretation of these instabilities. Some conclusions can be drawn directly from the observations made. Obviously, pellets create a strong local 3D perturbation evolving into a global one on the transport time scale. The 3D perturbation can initiate explosive evolution of ELMs, provided that the edge conditions allow mode growth. The seed perturbation appears so strong that it can restore fast ELM onset even in regimes characterized by slow spontaneous ELM growth. In the type-I regime with a hot (low-resistivity) edge, the spontaneous growth rate seems to be already at the upper limit. Even imposing the strong seed perturbation cannot raise it further. In the QH mode, the pellet perturbation seems to cause an initial destabilization, but the ambient plasma does not follow and the mode decays away without causing a major impact.

The ability to trigger any MHD mode by providing suitable seed perturbations seems to be a general feature for pellets. However, this may not necessarily be due to local, prompt, 3D perturbation, but from the global impact on plasma density and temperature profiles.

All the above states of affairs appear, however, consistent with the conjecture that the plasma, at the moment of impact of the pellet, is in a nonlinearly unstable state or in one-as in the case of the snakewhere a stable, helical equilibrium also exists. The pellet causes a finite amplitude perturbation, which forms the seed of the rapid unstable development, or drives the transition into the adjacent helical state. This 
hypothesis, which is consistent with the theoretical picture outlined in [10], will be followed up by further experiments during the upcoming campaign. In this picture, the failure of the pellet to trigger a full-blown ELM in the quiescent $\mathrm{H}$-mode regime would be interpreted as indication that, in this case, edge plasma profiles are kept well below the instability threshold by the EHO-induced transport.

\section{REFERENCES}

1. P. T. Lang, J. Neuhauser, L. D. Horton, et al., Nucl. Fusion 43, 1110 (2003).

2. ASDEX Upgrade Team, Fusion Sci. Technol. 44, 569 (2003).
3. G. Federici, A. Loarte, and G. Strohmayer, Plasma Phys. Controlled Fusion 45, 1523 (2003).

4. G. Kocsis, S. Kalvin, P. T. Lang, et al., Nucl. Fusion 47, 1166 (2007).

5. A. Kallenbach, P. T. Lang, R. Dux, et al., J. Nucl. Mater. 337-339, 732 (2005).

6. H. Zohm, Plasma Phys. Controlled Fusion 38, 105 (1996).

7. W. Suttrop, V. Hynonen, T. Kurki-Suonio, et al., Nucl. Fusion 45, 721 (2005).

8. H. Zohm, G. Gantenbein, A. Gude, et al., Nucl. Fusion 41, 197 (2001).

9. J. A. Wesson, Plasma Phys. Controlled Fusion 37, A337 (1995).

10. H. Wilson and S. Cowley, Phys. Rev. Lett. 92, 175006 (2004). 